Corresponding Author: Tri Hapsari Retno Agustiyowati; email:

agustiyowati60@gmail.com

Published: 7 February 2022

Publishing services provided by Knowledge E

(c) Tri Hapsari Retno

Agustiyowati and Sudirman

Sudirman. This article is

distributed under the terms of the Creative Commons

Attribution License, which permits unrestricted use and redistribution provided that the original author and source are credited.

Selection and Peer-review under the responsibility of the IVCN Conference Committee.

\section{The Psychological Experience of Nurses Carrying Out Hemodialysis While Caring for Patients With COVID-19 in Indonesia}

\author{
${ }^{1}$ Jurusan Keperawatan, Poltekkes Bandung \\ ${ }^{2}$ Jurusan Keperawatan Poltekkes Kemenkes Semarang \\ ORCID
}

Tri Hapsari Retno Agustiyowati1, ${ }^{1,}$ and Sudirman Sudirman ${ }^{2}$

Tri Hapsari Retno Agustiyowati: https://orcid.org/0000-0001-6126-3531

\begin{abstract}
Several studies have been conducted to assess the impact of the COVID-19 pandemic on mental health. However, few studies have been conducted in Indonesia to investigate the impact of the pandemic on the psychological experiences of nurses in carrying out hemodialysis (HD) while caring for patients with COVID-19 in Indonesia. Thus, this was the purpose of this study. The Colaizzi phenomenological approach was applied. This study included 20 HD nurses. Qualitative approaches were used to provide an overview of the nurses' psychological state during the pandemic. This study identified three primary themes: dealing with uncertainty, personal care and protection from COVID-19, and psychological resilience. The HD nurses were affected by the pandemic, and this study's findings can be utilized by policymakers to make judgments about how to respond. The results may also help health workers and volunteers recognize mental health issues and develop mental health rehabilitation programs for HD nurses affected by the COVID-19 pandemic.
\end{abstract}

Keywords: COVID-19, hemodialysis, live experience, nurse, psychological

\section{Introduction}

Nurses play a vital part in the fight against the epidemic in every country. The International Council of Nurses (ICN) established a Disaster Care Competencies framework in 2009 that includes ten skill domains for disaster nurses [1]. However, nurse's well-being and safety are critical not just for their own sake, but also for the sake of their patients' safety and the prevention of epidemics [2]. By May 2020, almost 90,000 nurses had verified COVID-19, and over 260 had perished [1]. As of June 2020, there were 61 confirmed cases of COVID-19 in Indonesian nurses, 97 cases of COVID-19 positive without symptoms, 48 cases of rigorous surveillance, and 19 deaths (INNA, 2020).

Frontline healthcare personnel who are actively involved in the diagnosis, treatment, and care of heamodyalisis (HD) patients with COVID-19 are at risk of developing psycho- 
scenario. The increasing number of confirmed and suspected cases, demanding workloads, depletion of personal protective equipment, broad media coverage, a lack of specific drugs, and feelings of inadequate support can all contribute to the mental strain on these health care personnel. Previous research has found that health-care employees experienced negative psychological effects as a result of the 2003 SARS outbreak [3-6]. Health workers who are concerned about the spread of disease to their loved ones and coworkers are more likely to avoid the workplace or consider retiring, according to a study by $[3,4]$. They also report experiencing symptoms of stress, high levels of anxiety, and depression that can have long-term psychological consequences. Concerns regarding mental health, psychological adjustment, and healing are also prominent. Health care workers who are caring for COVID-19-infected HD patients are now becoming available.

Nursing in Indonesia during the early stages of the COVID-19 pandemic is unique. The public refused to bury several of the COVID-19-dead nurses. Additionally, a nurse who is known to treat COVID-19 patients is not permitted to return home due to widespread fears of disease transmission. COVID-19 is a new disease with diverse healthcare systems and cultures, hence further research is needed on the psychological experiences of HD nurses caring for COVID-19 patients. Research on the experiences of HD nurses who care for patients with COVID-19 in Indonesia has not yet been published in Indonesia. As a result, our study sought to understand the psychological experiences of HD nurses who were treating HD patients with COVID-19 in Indonesia.

\section{Methods}

\subsection{Study design}

The Colaizzi phenomenological technique is used in this study to qualitatively assess the psychological experiences of HD nurses who treat HD patients with COVID-19. Colaizzi's phenomenological method focuses on participants' experiences and feelings, looking for shared patterns rather than unique traits in research subjects. This scientific approach ensures the authenticity of the experiences gathered by participants in order to meet scientific standards. From March to May 2021, this study will be done at multiple COVID-19 referral hospitals in Bandung. 


\subsection{Sample}

We used a purposive sampling strategy to identify $20 \mathrm{HD}$ nurses who treated HD patients with COVID-19. HD nurses who were members of the COVID-19 team and provided nursing care to HD patients with verified COVID-19 met the inclusion criteria. The number of respondents required was established by interviewing HD nurses who fit the inclusion criteria until the data was saturated and no new issues were produced.

\subsection{Guidelines for interviews}

The interview framework was developed by a review of the relevant literature, consultation with experts, and the selection of two nurses for pre-interviews. The following were the key interview questions posed to participants: (1) What are the basic psychological feelings of nursing care providers for COVID-19 HD patients? (2) What is your coping mechanism? (3) What are your thoughts on how to cope with the epidemic? We also asked the following sub-questions: (1) How did you feel when you were assigned the anti-epidemic task? (2) How do you feel when you use COVID-19 to treat HD patients? (3) How has your life changed? (4) How do you deal with changes in your professional and personal life? (5) What are your ideas and thoughts about this anti-epidemic task?

\subsection{Procedure}

Face-to-face interviews were performed in different rooms in complete silence. The interviews were taped and kept private. Interviews last between 40 and 60 minutes each person. If participants displayed emotional issues during the interview, appropriate psychological help was provided to avoid secondary psychological damage. Subjects were free to withdraw their permission at any moment. Researchers maintained neutrality while gathering data and developing positive relationships with subjects. To increase data authenticity and avoid bias, we employ approaches such as unconditional acceptance, active listening, and clarification. At least 1 - 2 face-to-face interviews and 1 - 2 telephone interviews were scheduled for each participant as needed to assure data collection at different time points. 


\subsection{Data analysis}

The recordings were transcribed and evaluated using the Colaizzi phenomenological analysis approach within 24 hours of each interview. Two researchers analyzed the interview materials separately, summarized and extracted important statements, and developed the themes. The study group, which included a nursing master, a nursing doctor, and two chief nurses, discussed and settled conflicting ideas about the theme's content.

\section{Results}

As stated in Table 6.1, the sample for this investigation comprised of 20 nurses. The participants' average age was 34.6 years (standard deviation (SD) 9.78 years), and they had 10.1 years of job experience (SD 4.7 years). Everyone in the group was married. 14 of the 20 competitors were female. Participants were from a variety of disciplines, with a total of nine individuals from government hospital hemodialysis units and 11 participants from private hospital hemodialysis units. No one has ever worked in a large-scale epidemic like the current COVID-19.

\subsection{Theme 1: Difficulties in dealing with unknown conditions}

Nurses working in COVID-19 facilities encounter numerous problems that impair their physical, psychological, and emotional well-being. This subject is divided into four categories: working in a new situation, heavy workload, discomfort wearing PPE, and dread of COVID-19. None of the nurses in the study had previously worked in a pandemic situation such as the present COVID-19 epidemic. Nurses must adjust to new policies and career paths. Most nurses noted that the abrupt change in roles and responsibilities, along with the fact that the virus was not well understood, caused a lot of stress:

"This is a new disease, and we don't know anything about it." We must take extra measures, learn new medications, and follow new protocols. This is our first time doing something like this." (R2)

"For the first three weeks, I was allocated to COVID-19 patients. As the severity of the cases increased, I was dispatched to the emergency room to treat critically ill patients. I was eventually transferred to the inpatient ward. You will be trained as a new member of staff." (N4) 
The frontline nurses were tired by the spike in patients during the early stages of the pandemic. Nurses must care for more patients than ever before while also adapting to rigorous shift schedules in order to meet staffing needs. They have more responsibilities to do and must work long hours to provide crucial care to sick patients. The majority of nurses are exhausted as a result of their workload:

"Look at this rush of patients." The first two months were challenging. I began to experience anxiety episodes and sleepless nights. I'm constantly thinking about work. "I recall everything that happened during the day, whether I did everything correctly, gave the correct prescription, or missed anything." (R5)

Full personal protection equipment (PPE). Wearing PPE, according to the nurses in the study, was extremely uncomfortable. Nurses were suffocating and sweating due to the airtightness of the N95 masks and bulky gowns. Some nurses report headaches and pressure injuries to their faces as a result of long-term use. Furthermore, wearing complete PPE creates a barrier to basic human needs like as feeding and elimination. Several participants emphasized the scarcity of PPE and the inconsistency of PPE quality:

"Wearing PPE is a problem, you have to wear and remove it in a certain way." Breathing, moving, and communicating are all difficult. All those layers are also making me sweat. Taking off PPE to drink was inconvenient. I recall having to keep my N95 for three days. That will suffice for the time being till supplies arrive." (N7)

Participants were afraid about getting COVID-19 because of its highly contagious nature and a lack of knowledge. COVID-19. The majority of individuals are concerned about spreading illnesses to their families: "I am concerned about contracting the virus." We don't know anything about it except that it's incredibly contagious and people are dying from it." (R.6)

"I was terrified l'd spread the infection to them." Unfortunately, I don't have anywhere else to stay, so l'm forced to sequester myself at home." (N.9)

\subsection{Theme 2: Personal care and COVID-19 protection}

During the epidemic, frontline nurses encounter numerous physical, psychological, and emotional obstacles as a result of COVID-19. This subject depicts the many coping techniques or modifications employed by nurses in this study to deal with the challenges of working in designated COVID-19 institutions. Nurses are concerned about getting COVID-19 and, as a result, spreading it to their families. Despite utilizing PPE and adhering to infection control protocols, nurses feel insecure due to frequent contact 
with patients infected with COVID-19. As a result, nurses felt compelled to take extra precautions to protect themselves and their families, something they would not have done under normal conditions. These actions are motivated by the nurse's intuition rather than infection control policies or regulatory agency recommendations. Nurses frequently wash and wear additional PPE over masks, gowns, and gloves to alleviate their concern of contamination. Some of the participants also were strangely preoccupied with cleaning up whatever objects they may have brought from the hospital as well as their cars.

"We began using disposable dishes, and I was required to wear a "special uniform" (a new set of scrubs and shoes for COVID use only). I would call home before entering the house after the job and segregate the kids in one room while I cleaned myself up." (R.10)

"Sometimes, I use extra gloves and perform mask surgery while wearing my N95 mask." When I get in the automobile, I wipe rubbing alcohol on my hands and then clean with alcohol." (R.3)

This subject illustrates how nurses' eating habits have changed in response to COVID19. Some of the nurses in the study ate more as a stress response, while others believed that eating more would enhance their antibodies to the virus:

"I eat a lot during the crisis." I suppose I gained roughly ten kilograms. I'm exhausted after work; I eat a lot so that I can have a lot of energy." (R9)

"I didn't eat well at initially, but I was able to maintain a good diet and exercise program during the COVID-19 pandemic." (N7)

During their battle with COVID-19, the nurses in this study sought social support. Nurses in COVID-19 facilities have the support of their families and the people they work with in a new world where social gatherings are prohibited:

"The best stress relief is talking to someone." Because of the pandemic, there is no way to visit friends or family." (R8)

\subsection{The third theme: psychological resilience}

Despite the risks involved, the nurses in this study displayed resilience in working in a pandemic. During this pandemic, nurses believe that their duty is the most vital. They find meaning and purpose in times of need, and it is part of their job. COVID-19 was viewed as an incentive to pursue by nurses in this study, rather than a reason to leave. 
"I felt as if I were on a mission." Some of my friends continue to whine about the restrictions, but I go to work." (R8)

"I believe it is our responsibility as nurses to care for them. I never considered quitting. Being a nurse is fantastic because it allows you to serve people regardless of who they are or where they come from." (R1)

\section{Discussion}

The increase in patients during the pandemic is posing a significant strain to the healthcare workforce. Because of the growing number of COVID-19 patients, nurses are being deployed and sent to units outside of their experience and nursing background. As a result, they must adjust to new policies, report to new managers, collaborate with new coworkers, become familiar with the physical layout, and master new procedures. Nurses must quickly acclimate to this new circumstance. Nurses are stressed as a result of the abrupt change in roles and responsibilities. Adapting to a new work environment and experience is connected with emotions of despair and anxiety among healthcare practitioners, according to [7]. Despite attempts to prepare nurses for the pandemic, continual education and training should be provided to ensure that nurses are confident in their ability to care for patients during the epidemic [7]. Even before COVID-19, nurses were at a significant risk of having a negative quality of life professional experience due to stress and burnout [8].

Furthermore, wearing PPE for an extended period of time creates physical discomfort in nurses. Some nurses also questioned the quality of the hospital's PPE. Handling COVID-19 patients and adhering to infection control procedures increases nurses' workload, including procedures for putting on and removing PPE as well as the intensive care workload required by critically ill COVID-19, such as intubation, insertion of central and arterial lines, changes in position, and so on [9]. Furthermore, nurses must wear full PPE (head cover, N95 mask, face shield or goggles, gown, and shoe cover) for several hours to ensure their safety, particularly during aerosol-generating procedures. In this study, nurses discovered that wearing PPE for lengthy periods of time was uncomfortable and was connected with perspiration, headaches, suffocation, and facial ulcers. This finding is similar with the findings of a recent study that shows the difficulties that healthcare staff confront when managing COVID-19 patients while wearing PPE. Wearing a protective mask and case of clothing, for example, was rated as a major stressor by nurses. 
Despite the risks involved, the nurses in this study displayed resilience in working in a pandemic. During this pandemic, nurses believe that their duty is the most vital. They find meaning and purpose in times of need, and it is part of their job. COVID-19 was viewed as an incentive to pursue by nurses in this study, rather than a reason to leave. The ability to mentally or emotionally overcome a crisis or quickly recover to pre-crisis position is referred to as psychological resilience [10]. Resilience has been noted as a crucial aspect for those working in the nursing profession due to the high stress situations. Nurses experience a variety of issues that might lead to exhaustion or physical or mental diseases as a result of overwork or stress [11-13]. Resilience is a protective factor that alters, improves, or adjusts a person's response to a variety of environmental dangers that have an impact on maladaptive consequences [14]. Currently, no psychological therapies aimed at overcoming the psychological crises of nurses following the pandemic have been developed or used in Indonesia. This could be because COVID-19 has a significant risk of rapid human-to-human transmission, making face-to-face intervention very impossible $[15,16]$. In this context, psychological therapies should be provided differently than those frequently utilized in natural catastrophes or pandemics [17-19].

\section{Conclusion}

This study has three primary themes: dealing with uncertainty, personal care and protection from covid-19, and psychological resilience. HD nurses in Indonesia are affected by the pandemic, and this study's findings can be utilized as fundamental data for policymakers to make judgments about how to respond. The results of this study may also help health workers and volunteers recognize mental health issues and develop mental health rehabilitation programs for HD nurses affected by the COVID-19 epidemic.

\section{Conflict of interest}

All authors declare no conflict of interest

\section{Acknowledgment}

This study was funded by Poltekkes Bandung 


\section{References}

[1] International Council of Nurses. ICN COVID-19 portal. International Council of Nurses; 2020. Available from: https://www.2020yearofthenurse.org/

[2] Chang D, Xu H, Rebaza A, Sharma L, Dela Cruz CS. Protecting health-care workers from subclinical coronavirus infection. The Lancet - Respiratory medicine. 2020;8:13.

[3] Maunder R, Hunter J, Vincent L, et al. The immediate psychological and occupational impact of the 2003 SARS outbreak in a teaching hospital. Can Med Assoc J I'Association Medicale. Can. 2003;168(10):1245-51.

[4] Bai Y, Lin C-C, Lin C-Y, Chen J-Y, Chue C-M, Chou P. Survey of stress reactions among health care workers involved with the SARS outbreak. Psychiatri Service. 2004;55(9):1055-7.

[5] Lee AM, Wong JGWS, McAlonan GM, et al. Stress and psychological distress among SARS survivors 1 year after the outbreak. The Canadian Journal of Psychiatry. 2007;52(4):233-40.

[6] Chua SE, Cheung V, Cheung C, et al. Psychological effects of the SARS outbreak in Hong Kong on high-risk health care workers. The Canadian Journal of Psychiatry. 2004;49(6):391-3.

[7] Liu Q, Luo D, Haase JE, et al. The experiences of health-care providers during the COVID-19 crisis in China: A qualitative study. Lancet Glob Health. 2020;8(6):790-8.

[8] Kim Y. Nurses' experiences of care for patients with Middle East respiratory syndrome-coronavirus in South Korea. American journal of infection control. 2018;46(7):781-7.

[9] Franceschi C, Garagnani P, Parini P, Giuliani C, Santoro A. Inflammaging: A new immune-metabolic viewpoint for age-related diseases. Nature Reviews Endocrinology. 2018;14(10):576-90.

[10] Munoz R, Brady S, Brown V. The psychology of resilience: A model of the relationship of locus of control to hope among survivors of intimate partner violence. Traumatology (Tallahass Fla). 2016;3:23.

[11] Swanson WC, Carbon JB. Crisis intervention: Theory and technique. Task Force Rep Am Psychiatr Assoc Treat Psychiatr Disord. 1989.

[12] Everly GS, Lating JM, Gravitz MA. A clinical guide to the treatment of the human stress response. Springer; 2002.

[13] Yeager K, Roberts A. Differentiating among stress, acute stress disorder, crisis episodes, trauma, and PTSD: Paradigm and treatment goals. Brief Treatment \& Crisis Intervention. 2003;1:3. 
[14] He Z, Chen J, Pan K, et al. The development of the. Int J Biol Sci.

[15] Yang Y, Peng F, Wang R, et al. The deadly coronaviruses: The 2003 SARS pandemic and the 2020 novel coronavirus epidemic in China. Journal of autoimmunity. 2020;109:102434.

[16] Wee LE, Conceicao EP, Sim XYJ, et al. Minimizing intra-hospital transmission of COVID-19: The role of social distancing. Journal of Hospital Infection. 2020;105(2):113-5.

[17] Petzold MB, Plag J, Ströhle A. Dealing with psychological distress by healthcare professionals during the COVID-19 pandemic. Nervenarzt. 2020;91(5):417-21.

[18] Nan Z. Psychological and behavioral impact of Wuhan lockdown and suggestions. authors. 2020;35(3).

[19] Chen Q, Liang M, Li Y, et al. Mental health care for medical staff in China during the COVID-19 outbreak. The Lancet-Psychiatry. 2020;7:15-6. 